\title{
Development of a reference Phasor Measurement Unit (PMU) for the monitoring and control of grid stability and quality
}

\author{
Hervé Ndilimabaka ${ }^{1}$, Isabelle Blanc ${ }^{1}$ \\ ${ }^{1}$ Laboratoire National de Métrologie et d’Essais, 29 Avenue Roger Hennequin, 78197 Trappes, France
}

\begin{abstract}
This paper discusses the details of the development of a Phasor Measurement Unit regarding the requirements of the IEEE C37.118-2005 synchrophasor standard relative to steady-state conditions on grid monitoring and control. This phasor measurement unit is intended to be used for field tests sooner.
\end{abstract}

\section{Introduction}

The growing concern worldwide for both climate change and environmental matters forces governments to make strong decisions on how to produce, consume, and store energy. More specifically in the framework of electricity, these actions focus on the transformation of our present electricity grids into smart grids, a step crucial for the successful uptake of renewable energy sources into the grid while reducing in the same time carbon dioxide emissions in the atmosphere.

A smart electricity grid can be thought as the superposition of a traditional electricity grid (the attention is more focused here on the distribution grid compared to both production and transport grids as the former is more concerned with the uptake of renewable energy sources) with a communication structure capable of help maintaining the stability, the reliability and the quality of the grid in a context of interconnecting wide-area electricity networks.

One useful tool for achieving this functionality is the phasor measurement unit (PMU), which takes time synchronized to the measurements of voltage and current signals on a power grid and derive some relevant quantities in relation with the grid state. PMUs applications have gained worldwide acceptance for the monitoring, protection and control of wide-area electricity networks $[1,2]$. However most large PMU implementations for grid monitoring use devices from various manufacturers, involving then different measurement technologies and accuracies. This is due to a lack of a metrology framework surrounding the deployment of smart grid solutions [3]. The combined effort of 18 European national metrology institutes and 4 universities and research centres is currently focusing on the development of the necessary metrology tools to facilitate the observability and controllability of the smart grid technology.

\footnotetext{
${ }^{a}$ Hervé Ndilimabaka: Herve.Ndilimabaka@lne.fr
}

Here we give an overview of the development of a reference PMU according to the relevant quantities in terms of monitoring and control of the electricity grid, as defined in the IEEE C37.118-2005 standard [4]. The reference PMU hardware architecture, inspired by NIST realization [5] and its software implementation under LabVIEW environment are explicated.

\section{Development of the reference PMU}

The developed tool was built in two steps: hardware and software architectures. Even though this development was made in order to cover adequately the steady-state characterization according to the IEEE C37.118-2005 synchrophasor standard, the modules implemented could deal with dynamic characterization owing to some algorithms improvements. In such a case, no additional change would be needed concerning the hardware architecture.

\subsection{PMU hardware architecture}

The hardware architecture was derived from relevant existing literature on the subject from NIST [5,6]. The different modules were chosen in order to cover the requirements for field tests regarding transportability, ruggedness and immunity to external disturbances.

Figure 1 presents this hardware architecture. It contains four main blocks listed as follows:

- a signal conditioning block, which consists in the interface with the electricity grids through current and voltage transformers (at the upper part of the block) and signal conditioning capabilities (at the lower part of the block). This last part consists in a NI SCB-68 shielded block in which anti-aliasing filters have been built.

- a data acquisition block containing high speed data acquisition converters (PXIe-6366 
multifunction DAQ with 8 simultaneous analog inputs for a maximum sampling rate of $2 \mathrm{MS} / \mathrm{s} / \mathrm{ch}$ ), intended to acquire simultaneously the three phases associated with the power grid and the neutral for both current and voltage.

- a timing and synchronization block, containing two modules: PXIe-6674T timing module, intended to offer a controllable $10 \mathrm{MHz}$ highstability oscillator and PXIe-6682H synchronization module, intended to monitor the time base according to GPS time or IRIG-B or IEEE 1588 signals. In a signal-based synchronization, clocks and triggers are physically connected between the systems and the highest-precision is achieved. But the distance over which this synchronization can be performed is limited. In a time-based synchronization however the system components have a common time reference, events, triggers and clocks can be generated based on this time reference, signals can be timestamped and correlated in post-processing analysis and the distance required is unlimited in the case where the GPS signal is used.

- LabVIEW real-time operating system (installed on the NI PXIe-8133 controller): this block deals with the signal processing algorithms and communication with the host computer.

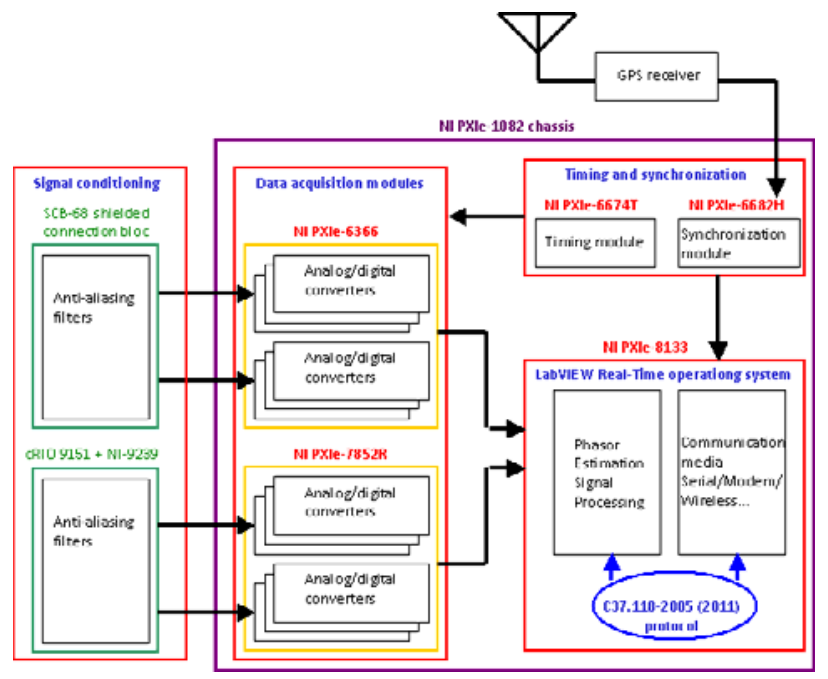

Figure1. Metrology grade reference PMU architecture.

Signal processing algorithms developed according to the IEEE C37.118-2005 standard are implemented into the software part of PMU architecture in order to derive PMUs parameters.

\subsection{PMU software architecture}

\subsubsection{Definition of a synchrophasor}

One major difference between PMUs and traditional supervisory control and data acquisition (SCADA) devices usually used for monitoring and control of the electrical grids lays in the synchronization of PMUs to the global positioning system (GPS) time. Doing this way allows the sampled data to be time stamped according to GPS. Data measured by different PMUs at various locations can then be compared to each other for state estimation (static and dynamic behaviour) of the grid.

Considering an input waveform represented mathematically by the following equation:

$$
x(t)=X_{m} \cos \left(2 \pi f_{0} t+\phi(t)\right)
$$

where $X_{m}$ stands for the magnitude of the sinusoidal waveform, $f_{0}$ the nominal frequency and $\phi(t)$ the instantaneous phase of the input waveform.

The phasor representation (figure 2) of such a waveform is correlated with the root mean square (rms) value of magnitude and the instantaneous phase as follows:

$$
\underline{X}=\frac{X_{m}}{\sqrt{2}} \exp (j \phi(t))
$$

which can be expressed in another manner as :

$$
\underline{X}=\underline{X_{r}}(t)+j \underline{X_{i}}(t)
$$

In this representation the instantaneous phase takes into account the possible variation of the grid frequency (the power system seldom operates at exactly the nominal frequency):

$$
\phi(t)=2 \pi \Delta f t+\phi_{0}
$$

which gives for the phasor:

$$
\underline{X}=\frac{X_{m}}{\sqrt{2}} \exp \left(j 2 \pi \Delta f t+\phi_{0}\right)
$$

A synchrophasor is then defined as the magnitude and angle of a cosine signal as referenced to an absolute point in time. This time being referenced to UTC time.

The IEEE C37.118-2005 standard focuses on steady-state signals (constant frequency over the period of measurement). Its revision (IEEE C37.118-2011) includes the dynamic aspects of PMUs measurements. 


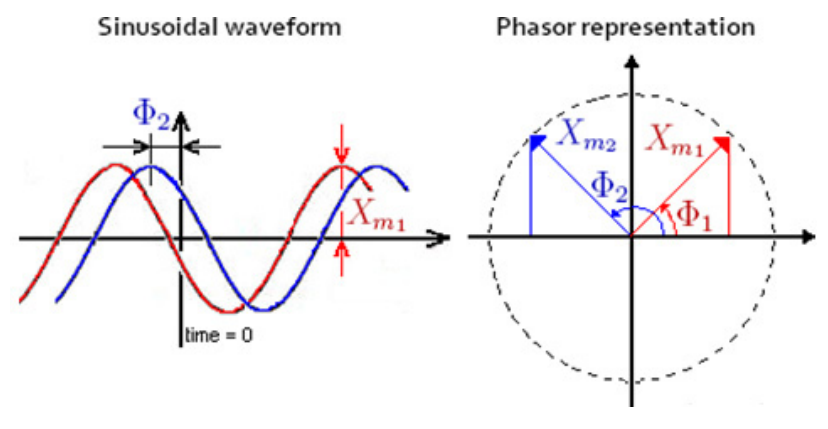

Figure2. Metrology grade reference PMU architecture.

PMUs parameters can then be calculated from the following equations:

$$
f(t)=\frac{1}{2 \pi} \frac{d \phi(t)}{d t}
$$

and its rate of change:

$$
R O C O F=\frac{d f(t)}{d t}
$$

According to the synchrophasor standard (IEEE C37.118-2005), the evaluation of the measurement quality from PMUs (magnitude, phase and frequency accuracy of PMUs) is made through the concepts of total vector error ( $T V E$ ), the error associated with frequency measurement (frequency error, $F E$ ) and the error coming from the evaluation of the rate of change of frequency ( $R F E$ ).

$T V E$ is defined by the root mean square of the difference between the mathematically defined theoretical phasor $\underline{X}$ and the estimated one (owing to PMU algorithms) $\underline{X}(n)$ as follows:

$$
T V E=\sqrt{\frac{\left(X_{r}(n)-X_{r}\right)^{2}+\left(X_{i}(n)-X_{i}\right)^{2}}{X_{r}^{2}+X_{i}^{2}}} \times 100 \%
$$

and $R F E$ and $F E$ by the following equations:

$$
F E=\left|f_{\text {theor. }}-f_{\text {meas. }}\right|
$$

or

$$
F E=\left|\Delta f_{\text {theor. }}-\Delta f_{\text {meas. }}\right|
$$

and for $R F E$ :

$$
R F E=\left|\left(\frac{d f}{d t}\right)_{\text {theor. }}-\left(\frac{d f}{d t}\right)_{\text {meas. }}\right|
$$

Compliance with IEEE C37.118-2005 synchrophasor standard requires a measurement difference within $0.1 \%$. Some efforts have been reported in literature [7, 8] to help developing the necessary tests in both steady state and dynamic conditions for the evaluation of PMUs performances. This latter point concerning the dynamic aspects of PMUs measurements is more correlated with the pending revision (IEEE C37.118-2011) of IEEE C37.118-2005 [4].

Another important difference between PMUs and traditional SCADA systems concerns the reporting rates of the reporting of PMU parameters (magnitude, frequency, rate of change of frequency, total vector error, frequency error, and rate of change of frequency error). The IEEE C37.118 synchrophasor standard proposes to standardize several reporting rates and reporting intervals. The proposed required reporting rates are shown in Table 1. A given reporting rate must evenly divide a one second interval into the specified number of sub-intervals depending on the system nominal frequency.

\begin{tabular}{|l|l|l|l|l|l|l|l|l|l|}
\hline $\begin{array}{l}\text { System } \\
\text { frequency }\end{array}$ & \multicolumn{2}{|l|}{$50 \mathrm{~Hz}$} & \multicolumn{6}{l|}{$60 \mathrm{~Hz}$} \\
\hline $\begin{array}{l}\text { Reporting } \\
\text { rates }\end{array}$ & 10 & 25 & 50 & 10 & 12 & 15 & 20 & 30 & 60 \\
\hline
\end{tabular}

Table 1. Synchrophasor reporting rates.

\subsubsection{Software architecture}

The software development is made under the LabVIEW Real-Time environment. The real-time operating system is necessary for the determinism (minimum jitter between each iteration) of each step and in order to have an autonomous system. As constructed, the PMU can be used as a stand-alone device capable of storing both raw and processed data or a device communicating with a remote computer, through an Ethernet connection.

Figure 3 represents the diagram of the developed system. Some relevant parameters are provided by the operator through the graphical user interface (GUI). These parameters comprise:

- the expected frequency of the grid $\left(f_{n}\right)$. The value of $f_{n}$ is in close relationship with the appropriate reporting rate $\left(F_{s}\right)$;

- the expected magnitude of the input waveform (here is depicted on figure 3 the voltage magnitude associated to one phase, $V_{n}$ );

- the expected phase (here the one associated with one of the voltage phases, phi_vn).

$f_{n}, V_{n}$ and phi_vn are used to synthesize the theoretical waveform from which are calculated the theoretical quantities for evaluating PMU parameters. 
To these parameters are associated the measurements parameters:

- $\quad$ the length of the acquisition window $\left(T_{a}\right)$;

- the number of samples before starting processing the acquired data $\left(N_{0}\right)$. This number permits to wait for a stable signal before starting processing of the acquired samples;

- the reporting rate $\left(F_{s}\right)$ which is selected according to Table 1 as mentioned above and its corollaries $F_{w}$ (number of frames per window) and $N_{f}$ (number of samples per frames).

From these parameters (signal parameters and measurement settings) some acquisition parameters are calculated:

- the sampling frequency $\left(f_{s}\right)$ owing to grid nominal frequency $\left(f_{n}\right)$, with respect to the Nyquist theorem;

- the number of samples acquired continuously $\left(N_{s}\right)$.

As defined, both acquisition and signal parameters allow, in association with the time-synchronization (phase-lock loop (PLL) with GPS time), to time stamp the acquired samples and generate the theoretical waveform owing to the waveform generator module. The acquired waveform contains information on the data points and the timestamps associated with.

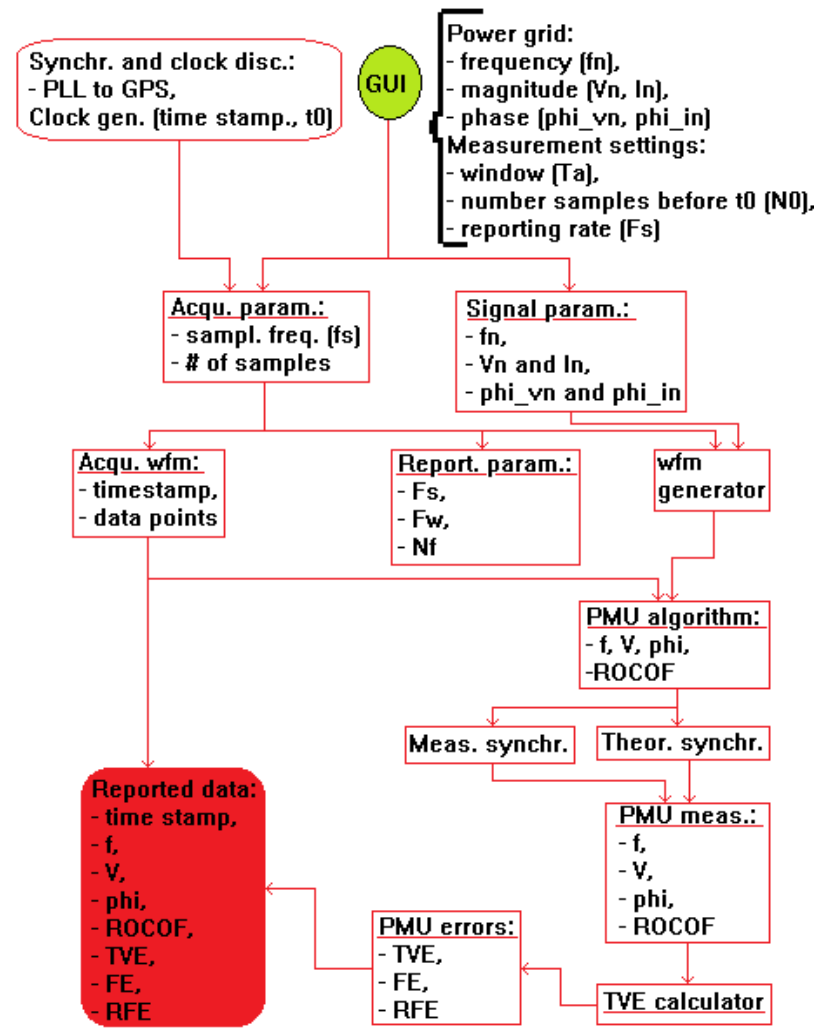

FIG.3. Reference PMU: software architecture.

Signal processing algorithms developed with Matlab and transcribed thereafter into LabVIEW owing to LabVIEW MathScript module, are used as PMU algorithms to derive the PMU parameters associated with the acquired waveform: frequency $(f)$, amplitude $(V)$, phase ( $p h i)$ and rate of change of frequency (ROCOF). These quantities are derived for both the synthesized waveform (theoretical synchrophasor) and the acquired waveform (measured synchrophasor).

The TVE calculator algorithm is then used for deriving PMUs errors (TVE, FE and RFE).

To this set of data $(f, V, p h i, R O C O F, T V E, F E$ and $R F E$ ) is associated the timestamp of the middle of the window over which all these calculations are performed. The length of this window being depending on the reporting frame. For example a reporting frame of 10 frames/s $(10 \mathrm{~Hz}$ for reporting the set of data) corresponds to a maximum window length of $100 \mathrm{~ms}$. And for a total measurement window of one second $\left(T_{a}=1 \mathrm{~s}\right)$, the figures mean reporting PMU data set at the maximum rate of 10 times.

Another constraint concerns the minimum number of cycles over which the algorithms are performed. For the PMU algorithm, this number is $2(40 \mathrm{~ms}$ for a $50 \mathrm{~Hz}$ system). All these constraints have to be taken into account for the selection of the appropriate window over which the calculation process is made (software selection, the only required selection for the operator from the graphical user interface being the window length $T_{a}$ ).

\section{Conclusion}

We emphasized this paper on the topic of phasor measurement unit (PMU), since it is an essential device for monitoring and control of grid stability and quality. We presented here some of the elements surrounding the development of a metrology grade PMU. Even though this step is important for the comprehension of the subject, it will be only a part of our presentation, since we will focus, in the final form, on the results associated with the characterization of our reference PMU regarding steady state conditions and explain the difference to those coming from field measurements using our reference PMU. The characterization tests and the associated results will be described elsewhere in a sooner report.

This report focuses only on the description of two key points concerning the development of the reference PMU: hardware and software architectures.

However, as one has might noticed, if the National Instruments modules used for the development of the reference PMU present interesting properties concerning high speed data acquisition, they exhibit limited input ranges $( \pm 10 \mathrm{~V})$. So one has to refer to the utilization of current and voltage transformers (CTs and VTs) in order to fit with real grid values. The following step in this process will consists in studying the influence of CTs and VTs on the uncertainties budget. We will then investigate their influence on the relevant PMU parameters (phasors, frequency, ROCOF, TVE, FE and RFE).

\section{Acknowledgements}


The EMRP is jointly funded by the EMRP participating countries within EURAMET and the European Union.

The research leading to these results has received funding from the European Union on the basis of Decision No. 912/2009/EC (Metrology for Smart Electrical Grids).

\section{References}

1. R. Moraes et al., Deploying a large-scale PMU system for the Brazilian interconnected power system. Electricity Utility Deregulation and Restructuring and Power Technologies, p. 143-149 (2008).

2. $\mathrm{Y}$. $\mathrm{Hu}$ et al., Progresses in PMU testing and calibration. Electricity Utility Deregulation and Restructuring and Power Technologies, p. 150-155 (2008).

3. "European Technology Platform Smart Grids Vision and Strategy for Europe's Electricity Networks of the Future", European Commission EUR 22040, 2006, http://ec.europa.eu/research/energy/pdf/smartgrids_en.pdf and on www.smartgrids.eu.

4. IEEE Standard for Synchrophasors for Power Systems, IEEE Standard C37.118-2005, March 2006.

5. G. Stenbakken, T. Nelson, Proc. Of the 41st Hawaii Conf. On Syst. Sci. (2008).

6. K. Narendra, Z. Zhang, J. Lane, B. Lackey, E. Khan, iREP Symp. Bulk Power Syst. Dynamics and Control (2007).

7. K. E. Martin and J. Stenbakken, "PMU System Testing and Calibration Guide", NASP Performance \& Standards Task Team (PSTT), 2007.

8. K. E. Martin, T. Faris, and J. Hauer, "Standardized Testing of Phasor Measurement Units", in Fault and Disturbance Analysis Conference Georgia Tech, Atlanta, GA, 2006. 